\title{
Coronal mass ejections as expanding force-free structures
}

\author{
Maxim Lyutikov and Konstantinos N. Gourgouliatos \\ Department of Physics, Purdue University, 525 Northwestern Avenue, West Lafayette, IN \\ 47907-2036
}

\begin{abstract}
We model solar coronal mass ejections (CMEs) as expanding force-free magnetic structures and find the self-similar dynamics of configurations with spatially constant $\alpha$, where $\mathbf{J}=\alpha \mathbf{B}$, in spherical and cylindrical geometries, expanding spheromaks and Lundquist fields respectively. The field structures remain forcefree, under the conventional non-relativistic assumption that the dynamical effects of the inductive electric fields can be neglected. While keeping the internal magnetic field structure of the stationary solutions, expansion leads to complicated internal velocities and rotation, caused by inductive electric fields. The structure depends only on overall radius $R(t)$ and rate of expansion $\dot{R}(t)$ measured at a given moment, and thus is applicable to arbitrary expansion laws. In case of cylindrical Lundquist fields, magnetic flux conservation requires that both axial and radial expansion proceed with equal rates. In accordance with observations, the model predicts that the maximum magnetic field is reached before the spacecraft reaches the geometric center of a CME.
\end{abstract}

\section{Introduction}

Expansion of magnetic clouds is one of the basic problems in space physics and geophysics, related to propagation of solar disturbances, the coronal mass ejections (CMEs) through interplanetary medium (Forbes et al. 2006). As the space craft passes through the cloud, the magnetic field strength is higher than average, the density is lower, the magnetic pressure inside the cloud greatly exceeds the ion thermal pressure and the magnetic field direction changes through the cloud (e.g. Burlaga et al. 1982).

There are two basic models of magnetic clouds: (i) magnetic flux ropes, still connected to the Sun at the distances of the Earth (Burlaga et al. 1981; Cargill et al. 1995; Chen and Garren 1993); (ii) disconnected entities of spherical topology, which in the simplest paradigm can be modeled as spheromaks (e.g. Ivanov and Kharshiladze 1985; Vandas, Fischer, and Geranios 1991). These are stationary solutions, while the CMEs expand while propagating in 
the Solar wind. Previous attempts to find a structure of expanding spherical configurations have led to breakdown of force-free condition through appearance of pressure and inertial effects (Low 1982; Ivanov et al. 1989; Vandas et al. 1997). In this paper we find the structure of expanding magnetic configurations that remain force-free. In the force-free approach the dynamics of the system is dominated by the magnetic field and any plasma pressure or inertia terms are negligible, for that reason we shall not include in this study the equations of continuity of the fluid and we shall focus only on the magnetic field and on the induced electric field.

The importance of spheromaks in spherical geometry (Chandrasekhar and Kendall 1957) and of Lundquist (1951) fields (in cylindrical geometry) as examples of clouds with selfcontained magnetic field stems from the fact that these solutions are, in some sense, the minimal energy states and thus are stable to many ideal and resistive instabilities. Woltjer (1958) and Taylor (1974) formulated the plasma relaxation principle, according to which plasma evolution conserves magnetic helicity and, if the magnetic field is the only important quantity, it evolves to a force-free state with constant $\alpha$, where $\mathbf{J}=\alpha \mathbf{B}$, where $\alpha$ has some similarities to thermodynamic temperature, so a relaxed state should have constant $\alpha$ (Bellan 2006).

\section{Expanding Spheromaks}

\subsection{Magnetic Field of a Spheromak}

Spheromaks are stationary force-free configurations of plasma satisfying the condition $\mathbf{J}=\alpha \mathbf{B}$ with spatially constant $\alpha$. They are solutions of Grad-Shafranov equation in spherical coordinates when the poloidal current is a linear function of the magnetic flux. Below we will use "the basic spheromak" solution, corresponding to axially symmetric dipolar-like fields (Chandrasekhar and Kendall 1957).

Given the magnetic flux function $P(r, \theta)$ and poloidal current function $I(P)$, the magnetic field is

$$
\mathbf{B}=\frac{\nabla P}{r \sin \theta} \times \mathbf{e}_{\phi}+\frac{2 I(P)}{r \sin \theta} \mathbf{e}_{\phi}
$$

When only magnetic force is present, the magnetic flux function satisfies the Grad-Shafranov equation

$$
\Delta^{*} P+4 I I^{\prime}=0
$$

where

$$
\Delta^{*}=\partial_{r}^{2}+\frac{\sin \theta}{r^{2}} \partial_{\theta} \frac{\partial_{\theta}}{\sin \theta}
$$


is the Grad-Shafranov operator. For linear dependence $I=\alpha P / 2$ with spatially constant $\alpha$, Equation (2) has separable solutions in term of spherical Bessel functions $j_{n}(\alpha r)$. The first order solutions are then

$$
\begin{aligned}
& P_{0}=B_{0} \frac{r}{\alpha} j_{1}(\alpha r) \sin ^{2} \theta, \\
& B_{r}=2 B_{0} \frac{j_{1}}{\alpha r} \cos \theta, \\
& B_{\theta}=-B_{0} \frac{j_{1}+\alpha r j_{1}^{\prime}}{\alpha r} \sin \theta, \\
& B_{\phi}=B_{0} j_{1} \sin \theta .
\end{aligned}
$$

The first order spherical Bessel function $j_{1}$ can be expressed in terms of elementary functions, $j_{1}(x)=\sin x / x^{2}-\cos x / x$. Parameter $\alpha$ is related to the size of a spheromak, defined by the surface where radial magnetic field is zero, solution of $j_{1}=0, R=C_{\alpha} / \alpha, C_{\alpha}=4.49$.

\subsection{Radial Expansion of a Spheromak}

We now study the self-similar, non-relativistic expansion of axially symmetric forcefree structures with spatially constant $\alpha$-parameter. Below we discuss a procedure that allows to derive non-relativistic, time-dependent force-free solutions from a given stationary force-free configuration. First, we note that parameter $\alpha$ has dimension of inverse length, and thus is related to overall size of the magnetic structure $R$. For expanding solutions we take $\alpha=\alpha(t)$, but still spatially independent. According to self-similar prescription, we take all radial dependence to be in a form $\propto r \alpha \propto r / R(t)$. The induction equation, $\nabla \times \mathbf{E}+\partial_{t} \mathbf{B}=0$, as well as magnetic magnetic flux conservation, require that in a timedependent case $P=\left(\alpha / \alpha_{0}\right)^{2} P_{0}$, where $\alpha_{0}$ is the value at some initial time and $P_{0}$ is a solution of a stationary force-free problem (e.g. given by Equation (4)). Next, we are looking for ideal solutions, where electric field is always perpendicular to magnetic field and thus can be written as $\mathbf{E}=-\mathbf{V} \times \mathbf{B}$. Since we are looking for radially self-similar solutions, $\mathbf{V}=V \mathbf{e}_{r}(c f$. Low 1982, and Equation (7)). This is the velocity of radial expansion. The drift velocity of the magnetic field lines is given by $\mathbf{v}=\mathbf{E} \times \mathbf{B} / B^{2}$ and is by definition perpendicular to the magnetic field lines. These two approaches give different values for $\mathbf{V}$ and $\mathbf{v}$. Using vector identities we can conclude that the difference between two velocities is a vector parallel to the magnetic field lines. Indeed

$$
\mathbf{v}=\frac{\mathbf{E} \times \mathbf{B}}{B^{2}}=-\frac{\mathbf{V} \times \mathbf{B}}{B^{2}} \times \mathbf{B}=\mathbf{V}-\frac{\mathbf{V} \cdot \mathbf{B}}{B^{2}} \mathbf{B} .
$$

There is a freedom of choice between these velocities without altering at all the force-free solution. Equilibria of this type, which contain also some matter however, do not allow 
transition from one velocity to the other without changing the dynamics of the problems just by adding a velocity component parallel to the magnetic field. In that case, an azimuthal component in the velocity will give rise to inertial terms in the force balance equation. In this study we focus in force-free equilibria where the dynamics of the system are dominated by the magnetic field and inertia is negligible. Choosing self-similar scaling, $V=-r \partial_{t} \ln \alpha$, we find for axially symmetric magnetic field, given by Equation (1), the following electric field

$$
\mathbf{E}=r \frac{\dot{\alpha}}{\alpha} \mathbf{e}_{r} \times \mathbf{B}
$$

where dot denotes differentiation with time. It follows from Equation (5) that:

$$
\mathbf{v}=\frac{\mathbf{B}\left(\mathbf{B} \cdot \mathbf{e}_{r}\right)-\mathbf{e}_{r} B^{2}}{B^{2}} r \partial_{t} \ln \alpha .
$$

In deriving the solution we neglected dynamical effects of electric field, in particular displacement current and electric charge density, so that the force-free condition involves only magnetic field and electric current, $\mathbf{J} \times \mathbf{B}=0$. This is indeed a reasonable assumption for the solar system where the velocities are non-relativistic.

For the basic spheromak solutions (4), the electromagnetic fields become

$$
\begin{aligned}
& B_{r}=2 B_{0} \alpha \frac{j_{1}}{\alpha_{0}^{2} r} \cos \theta, \\
& B_{\theta}=-B_{0} \alpha \frac{j_{1}+\alpha r j_{1}^{\prime}}{\alpha_{0}^{2} r} \sin \theta, \\
& B_{\phi}=B_{0} j_{1}\left(\frac{\alpha}{\alpha_{0}}\right)^{2} \sin \theta, \\
& E_{r}=0, \\
& E_{\theta}=-B_{0} \frac{\alpha \dot{\alpha}}{\alpha_{0}^{2}} r j_{1} \sin \theta=-B_{\phi} \frac{\dot{\alpha}}{\alpha} r \\
& E_{\phi}=-B_{0} \dot{\alpha} \frac{j_{1}+\alpha r j_{1}^{\prime}}{\alpha_{0}^{2}} \sin \theta=B_{\theta} \frac{\dot{\alpha}}{\alpha} r, \\
& \mathbf{E}=\left(\frac{\dot{\alpha}}{\alpha} r\right) \mathbf{e}_{r} \times \mathbf{B} .
\end{aligned}
$$

We stress that the fields remain force-free, $\mathbf{J} \times \mathbf{B}=0$.

\subsection{Velocity Structure of the Solution}

Expanding force-free spheromaks have complicated internal velocity structure. At each point there is a well defined electromagnetic velocity $\mathbf{v}=\mathbf{E} \times \mathbf{B} / B^{2}$, normalised to $c$, 
Equation (7). The solution can be parametrized by the velocity of the expansion of the boundary, which is $v_{\theta}=v_{\phi}=0$ and $v_{r}=R(t) \partial_{t} \ln (1 / \alpha)=v_{0}$, see Figures 1.2$] 3$.

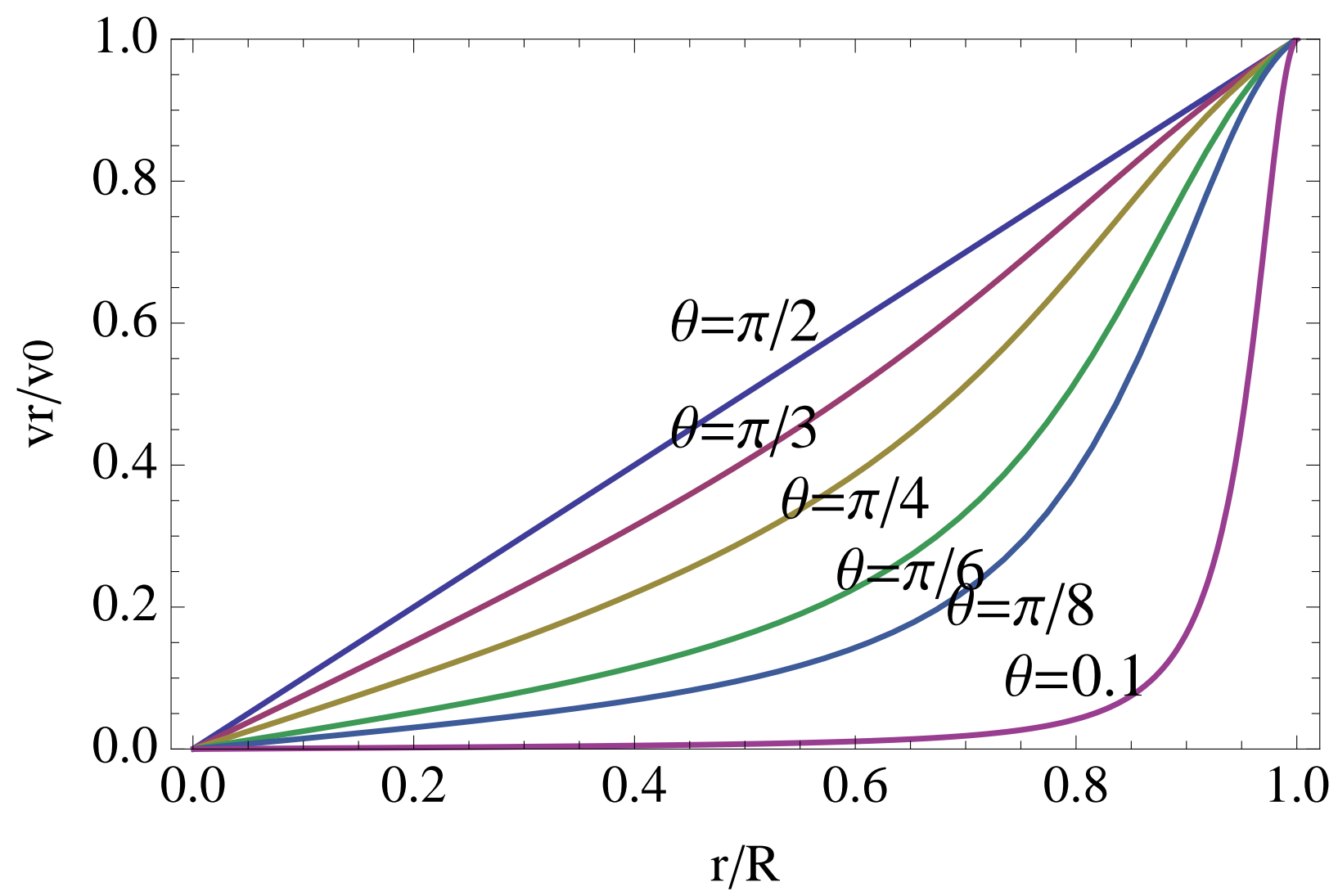

Fig. 1.- Radial velocity $v_{r}, v_{\theta}, v_{\phi}$ as functions of $r$ for different $\theta$. In the equatorial plane radial velocity increases linearly with radius, $v_{r}(\theta=\pi / 2)=(r / R) v_{0}$, while close to the axis velocity remains small in the bulk, sharply increasing to $v_{0}$ near the surface.

Since $v_{\phi} \neq 0$, the expansion of a spheromak induces rotation, with the different hemispheres rotating in the opposite direction (Figure 4). The maximum toroidal velocity is $0.33 v_{0}$ reached at $\theta=0.53$ and $r / R=0.71$. For $r \ll R$ the angular velocity of rotation is $\omega \sim \pm C_{\alpha} v_{0} \sin 2 \theta \frac{r}{4 R^{2}}$. The flow lines are plotted in Figure 5. Close to the symmetry axis, velocity at small radii is directed away from the axis.. Total velocity at small $r \ll R$ is $v \sim v_{0} r \sin \theta / R$. The flow lines are orthogonal to the surface, matching the overall expansion of the spheromak.

Using expressions (8) for electromagnetic fields, we can calculate the total energy of the 


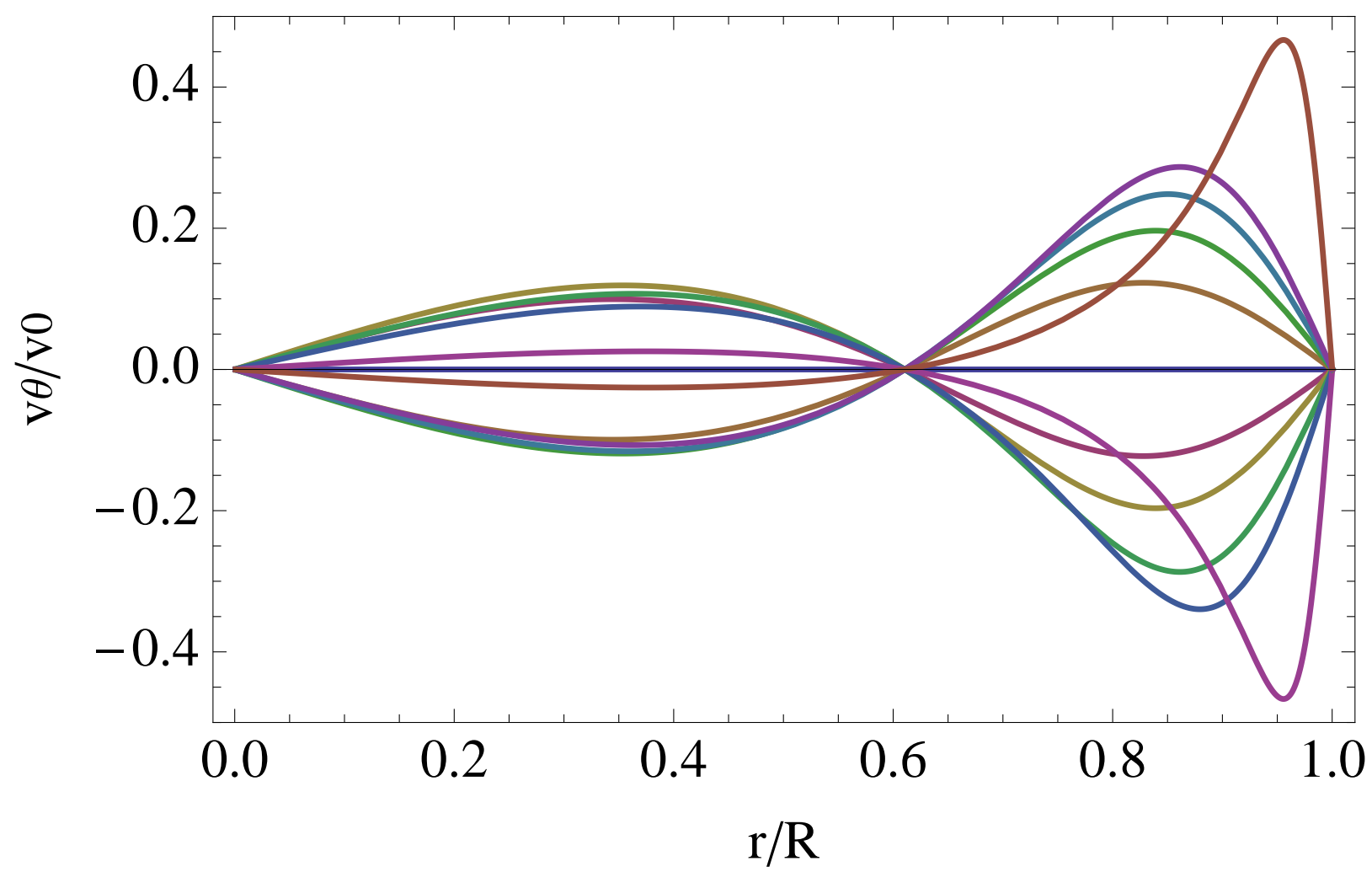

Fig. 2.- Velocity $v_{\theta}$ as a functions of $r$ for different $\theta$. Flows are antisymmetric with respect to the equatorial plane. $v_{\theta}$ becomes zero at $r=0.61 R$ (root of the equation $E_{\phi}=0$ )

expanding spheromak

$$
E_{\text {tot }}=\int d V \frac{E^{2}+B^{2}}{8 \pi}=\left(c_{1}+c_{2} \frac{v^{2}}{c^{2}}\right) \frac{B_{0}^{2} R_{0}^{4}}{R}
$$

where $c_{1}=2.5 \times 10^{-3}$ and $c_{2}=8.7 \times 10^{-4}$ are combinations of power laws and trigonometric functions of $C_{\alpha}$. The second term proportional to velocity squared is the contribution of the electric field and can be ignored for non-relativistic expansion.

It may be verified that the total magnetic helicity, $\mathcal{H}=\int d V \mathbf{B} \cdot \mathbf{A}=1.4 \times 10^{-2} B_{0}^{2} R_{0}^{4}$, as well as the toroidal magnetic flux $\mathcal{F}=\int B_{\phi} r d r=5.26 B_{0} R_{0}^{2}$ are independent of time. At the same time, the total electromagnetic energy decreases during expansion $\propto 1 / R$. This highlights an important point, that it is impossible to conserve both electromagnetic energy and magnetic helicity. In case of ideal expansion the energy decrease is due to $p d V$ work done on the surrounding plasma. Magnetic pressure on the surface $\propto 1 / R^{4}$, energy $\propto 1 / R$.

We can compare the self-similar spheromak solutions to the internal energy of adiabatically expanding polytropic gas with $p \propto \rho^{\gamma}, E \propto p V \propto R^{3(1-\gamma)}$. Thus, effectively, spheromak 


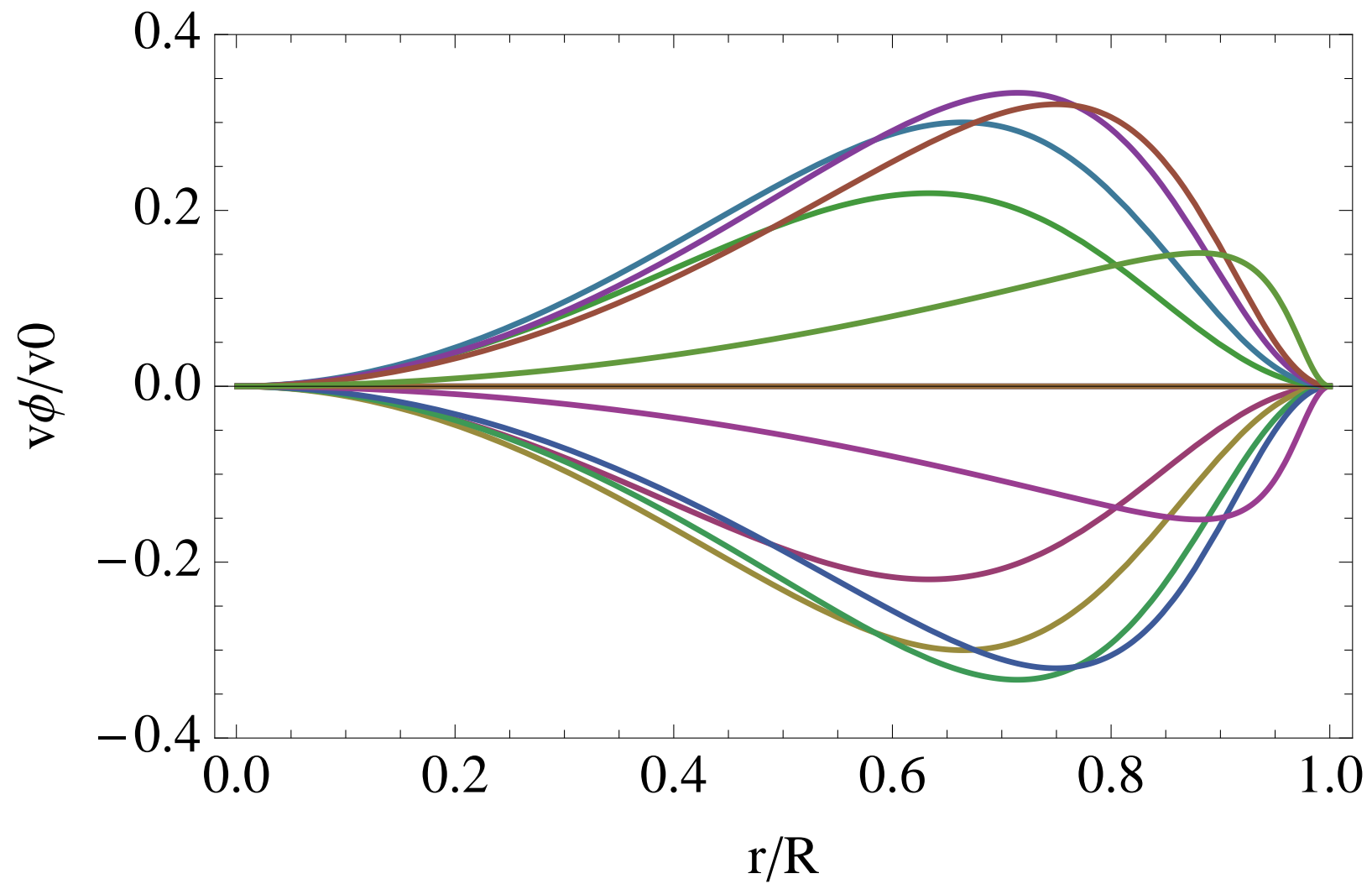

Fig. 3.- Velocities $v_{\phi}$ as functions of $r$ for different $\theta$. Flows are antisymmetric with respect to the equatorial plane.
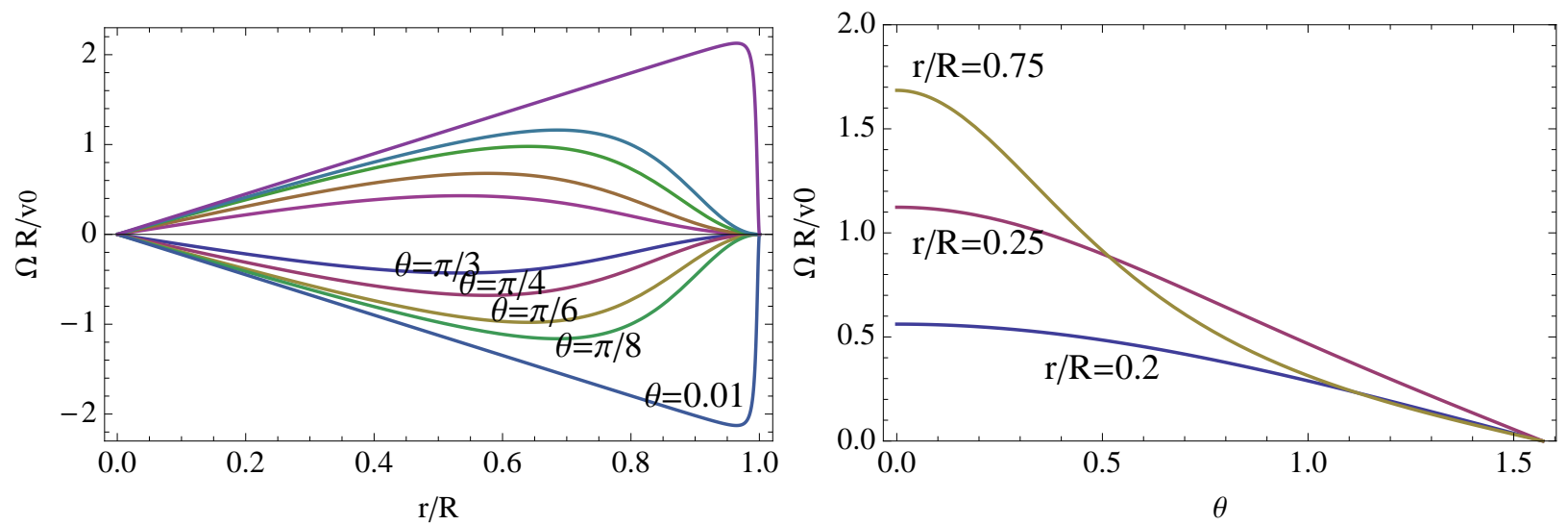

Fig. 4.- Angular velocity of rotation $\Omega=v_{\phi} /(r \sin \theta)$ as function of radius and polar angle.

behaves as a polytropic gas with $\gamma=4 / 3$. This is related to the results of Low (1982), who found self-similar MHD solutions for expanding cloud if the fluid obeys polytropic law with 


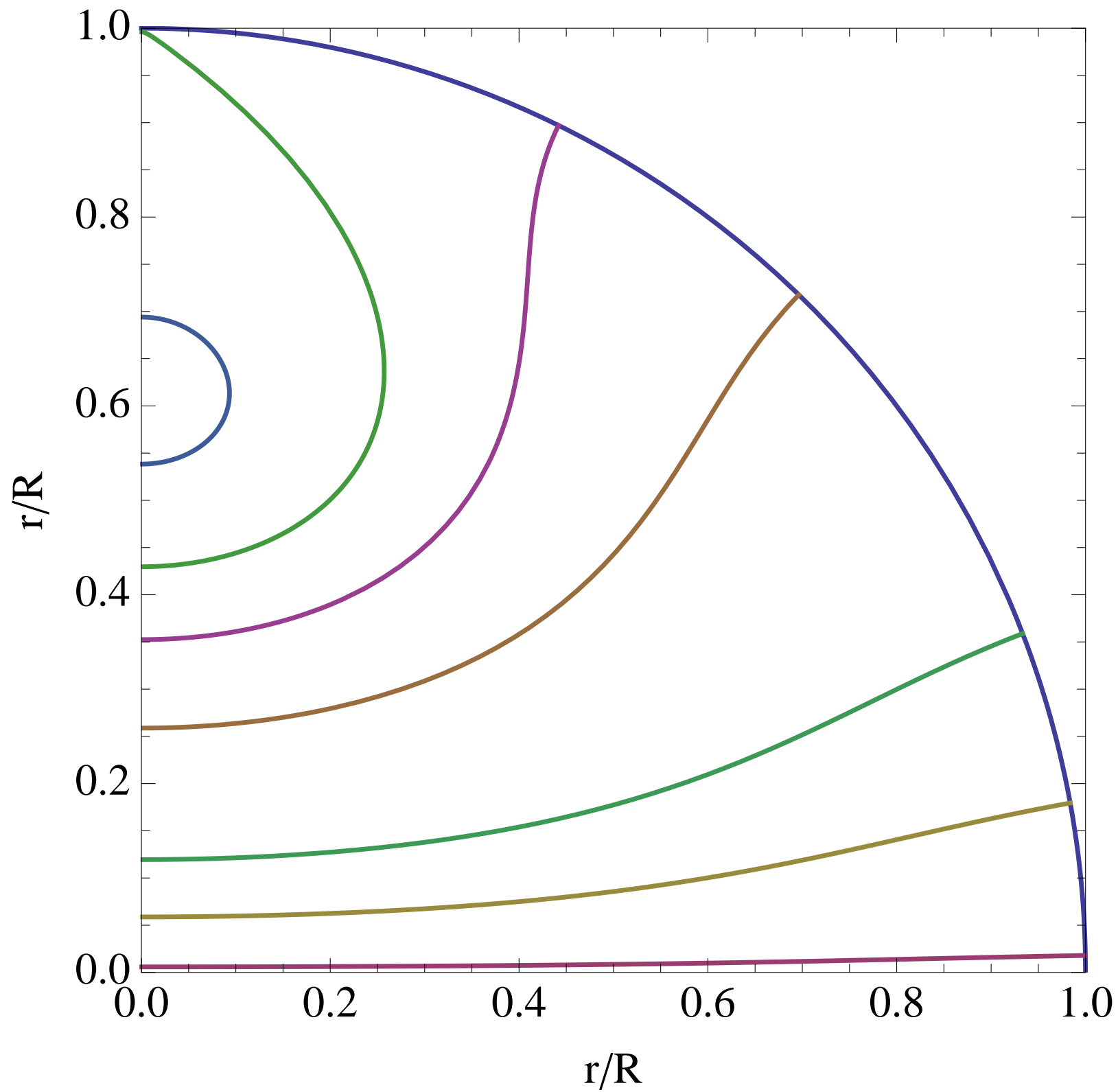

Fig. 5.- Plasma flow lines. On the surface flow lines become radial to match the overall radial expansion. The stationary point is $\theta=0, r=0.61 R$ (root of the equation $E_{\phi}=0$ ).

$\gamma=4 / 3$. In this case both magnetic and thermal components of plasma behave similarly (Gourgouliatos and Vlahakis 2010). 


\section{Toroidal Configurations}

In the case where magnetic clouds are connected to the Sun (Chen and Garren 1993), their topology shall resemble more an arcade, and their mathematical description is more accurately approximated by a force-free magnetic field confined in a torus. A number of studies (Burlaga et al. 1981; Burlaga 1988; Lepping, Burlaga, and Jones 1990) have considered solutions in cylindrical geometry as an approximation to the toroidal structures of magnetic cloud.

In this section we examine the effect of expansion in this type of solution by applying self-similarity. Let us assume a torus (Figure 6) of large radius $R_{0}$ and small $r_{0}$, the centre corresponding to the large radius is $O$ and the centre corresponding to the small radius lies on the circle $\left(O, R_{0}\right)$ and shall be called $O^{\prime}$. Let us attach an orthogonal system of coordinates to $O^{\prime}$; this coordinate system resembles a cylindrical coordinate system with the difference that the role of the axis $z$ is taken by the circle $\left(O, R_{0}\right)$, the coordinates in this system are $(\varpi, \phi, z)$, where $0 \leq \varpi \leq r_{0}, 0 \leq \phi \leq 2 \pi$ and $-\pi R_{0} \leq z \leq \pi R_{0}$; the field inside the torus has components $B_{\varpi}, B_{\phi}$ and $B_{z}$. Such fields have been studied by Miller and Turner (1981), who considered force-free structures in toroidal coordinates and found that for large aspect ratios $R_{0} / r_{0} \gg 1$ the solution reduces to Lundquist (1951) fields. The first few terms of the

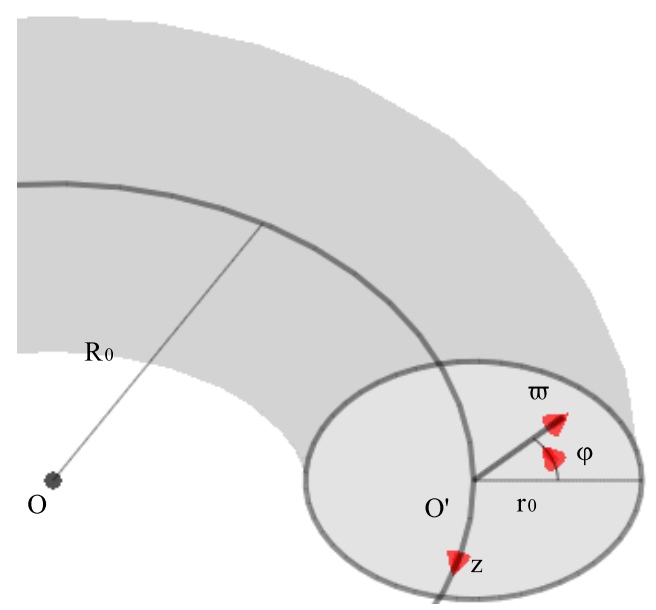

Fig. 6. - The geometry of the torus, the large radius $R_{0}$ writes a circle with centre $O$ and the small one $r_{0}$ a circle with centre $O^{\prime}$. We attach the orthogonal coordinate system $(\varpi, \phi, z)$ to the circle $\left(O^{\prime}, r_{0}\right)$.

Miller-Turner fields are

$$
B_{\varpi, \mathrm{T}}=\frac{B_{0}}{R_{0} \alpha}\left(-J_{0}(\alpha \varpi)+\frac{r_{0} F}{\varpi}\right) \sin \theta,
$$




$$
\begin{array}{r}
B_{\phi, \mathrm{T}}=B_{0}\left[J_{1}(\alpha \varpi)-\frac{1}{\alpha R_{0}}\left(J_{0}(\alpha \varpi)-r_{0} \frac{d F}{d \varpi}\right) \cos \theta\right], \\
B_{z, \mathrm{~T}}=B_{0}\left(J_{0}(\alpha \varpi)-\frac{r_{0}}{R_{0}} F \cos \theta\right), \\
F=\frac{\varpi}{2 r_{0}} J_{0}(\alpha \varpi)+\frac{1}{2} \frac{J_{0}\left(\alpha r_{0}\right)}{J_{1}\left(\alpha r_{0}\right)} J_{1}(\alpha \varpi) .
\end{array}
$$

Let us now consider a thin section of the torus which can be approximated with a cylinder. In the large aspect ratios limit, $R_{0} / r_{0} \gg 1$ we only keep the Lundquist field.

$$
\begin{array}{r}
B_{\varpi, \mathrm{S}}=0, \\
B_{\phi, \mathrm{S}}=B_{0} J_{1}(\alpha \varpi), \\
B_{z, \mathrm{~S}}=B_{0} J_{0}(\alpha \varpi),
\end{array}
$$

where $J_{0,1}$ is the Bessel function of the zeroth and first order.

Magnetic clouds expand as they propagate away from the Sun. The simultaneous expansion and propagation lead to an increase of both radii of the torus. The expansions of the small and large radii need to scale with the same factor to conserve the magnetic flux that crosses the equator of the torus and the magnetic flux that crosses a section of the torus: $\Phi_{1} \propto B_{\phi, \mathrm{T}} r_{0} R_{0}$ and $\Phi_{2} \propto B_{z, \mathrm{~T}} r_{0}^{2}$, so if $R_{0}$ is multiplied with some factor so does $r_{0}$.

This field shall experience the effect of expansion both in $\varpi$ and $z$ directions. Let us consider an expansion velocity $\mathbf{V}=-\varpi \frac{\dot{\alpha}}{\alpha} \hat{\mathbf{e}}_{\varpi}-z \frac{\dot{\alpha}}{\alpha} \hat{\mathbf{e}}_{z}$.

Similar to Section 2.2, the magnetic flux conservation of the expanding structure requires that

$$
\begin{array}{r}
B_{\varpi, \mathrm{E}}=0, \\
B_{\phi, \mathrm{E}}=B_{0}\left(\frac{\alpha}{\alpha_{0}}\right)^{2} J_{1}(\alpha \varpi), \\
B_{z, \mathrm{E}}=B_{0}\left(\frac{\alpha}{\alpha_{0}}\right)^{2} J_{0}(\alpha \varpi) .
\end{array}
$$

Then the electric field is $\mathbf{E}=-\mathbf{V} \times \mathbf{B}$, which gives

$$
\begin{gathered}
E_{\varpi}=-B_{0} \frac{\dot{\alpha} \alpha}{\alpha_{0}^{2}} J_{1}(\alpha \varpi) z, \\
E_{\phi}=-B_{0} \frac{\dot{\alpha} \alpha}{\alpha_{0}^{2}} J_{0}(\alpha \varpi) \varpi, \\
E_{z}=B_{0} \frac{\dot{\alpha} \alpha}{\alpha_{0}^{2}} J_{1}(\alpha \varpi) \varpi .
\end{gathered}
$$


The whole structure is force-free satisfying $(\nabla \times \mathbf{B}) \times \mathbf{B}=0$, indeed

$$
\nabla \times \mathbf{B}=B_{0} \frac{\alpha^{3}}{\alpha_{0}^{2}} J_{1}(\alpha \varpi) \hat{\mathbf{e}}_{\phi}+B_{0} \frac{\alpha^{3}}{\alpha_{0}^{2}} J_{0}(\alpha \varpi) \hat{\mathbf{e}}_{\mathbf{z}}=\alpha \mathbf{B} .
$$

In addition, the divergence of the magnetic field is zero $\nabla \cdot \mathbf{B}=0$ and the magnetic flux is conserved as it satisfies Faraday's law of induction $\nabla \times \mathbf{E}+\partial_{t} \mathbf{B}=0$, indeed

$$
\nabla \times \mathbf{E}=-B_{0} \frac{\dot{\alpha} \alpha}{\alpha_{0}}\left[\left(J_{1}(\alpha \varpi)+\alpha \varpi J_{0}(\alpha \varpi)\right) \hat{\mathbf{e}}_{\phi}-\left(\alpha \varpi J_{1}(\alpha \varpi)-2 J_{0}(\alpha \varpi)\right) \hat{\mathbf{e}}_{\mathbf{z}}\right]=-\partial_{t} \mathbf{B} .
$$

We stress that though we focus in on a thin slice of the torus area near $z=0$, where the $V_{z}$ component vanishes, we do take into account the fact that both the small and the large radii of the torus are increasing. The latter gives an expansion in the $z$ direction which creates a minor electric field in the $\varpi$ direction which is necessary to conserve the magnetic flux.

Again we evaluate drift velocity of the field lines $\mathbf{v}=\mathbf{E} \times \mathbf{B} / B^{2}$, whose components are

$$
\begin{array}{r}
v_{\varpi}=-\frac{\dot{\alpha}}{\alpha} \varpi, \\
v_{\phi}=\frac{\dot{\alpha}}{\alpha} \frac{J_{0}(\alpha \varpi) J_{1}(\alpha \varpi)}{J_{0}(\alpha \varpi)^{2}+J_{1}(\alpha \varpi)^{2}} z, \\
v_{z}=-\frac{\dot{\alpha}}{\alpha} \frac{J_{1}(\alpha \varpi)^{2}}{J_{0}(\alpha \varpi)^{2}+J_{1}(\alpha \varpi)^{2}} z .
\end{array}
$$

The components of $\mathbf{v}$ shall match $\mathbf{V}$ at the boundary. Indeed, the $v_{\varpi}$ component is mathced, then from the $\phi$ component we find that the cylinder shall end either at a root of $J_{1}$ or $J_{0}$ and then from the $z$ component we find the acceptable solution is that of a root of $J_{0}$, thus the end of the rope is defined by the position where $\alpha \varpi$ equals a root of $J_{0}$.

Formally, the $\phi$ and $z$ velocity components are proportional to $z$ and this breaks the translational symmetry of the Lundquist field. This is the outcome of choosing an arbitrary cross section and setting the velocity profile proportional to the distance from there. The physical system actually consists of a torus and as a result of the expansion an observer on a given point in the torus will see the other points to move away; and their distance measured on the $z$ coordinate will increase proportional to $z$. Given the arbitrary choice of the point $z=0$ we have the freedom to set this point at the place where the detector crosses the magnetic cloud, provided that its trajectory is perpendicular to the axis. The detector will 
see a structure where the values are given for $z=0$, the magnetic field is that of Equation (12), the electric field though is

$$
\begin{array}{r}
E_{\varpi, 0}=0, \\
E_{\phi, 0}=-B_{0} \frac{\dot{\alpha} \alpha}{\alpha_{0}^{2}} J_{0}(\alpha \varpi) \varpi, \\
E_{z, 0}=B_{0} \frac{\dot{\alpha} \alpha}{\alpha_{0}^{2}} J_{1}(\alpha \varpi) \varpi .
\end{array}
$$

Finally the velocity of the fluid is

$$
\begin{gathered}
v_{\varpi, 0}=-\frac{\dot{\alpha}}{\alpha} \varpi, \\
v_{\phi, 0}=v_{z, 0}=0 .
\end{gathered}
$$

We remark that similar results for the cylindrical Lundquist type fields have been obtained recently and independently by Dalakishvili et al. (2010).

\section{Matching to Driven Shock Solutions}

The magnetic clouds expand while propagating away from the Sun (Klein and Burlaga 1982). Next we consider how the expansion rate scales with the distance from the Sun. This depends both on the structure of the Solar wind and on the conservation or not of the magnetic flux and helicity of the cloud, during its evolution, which in turn is related to the connection or not of the cloud to the Sun. A spheromak-type cloud is a disconnected entity which ideally conserves its magnetic flux and helicity, however as it expands its electromagnetic energy cannot remain constant. Magnetic flux tubes, however, are attached to the Sun, so injection of magnetic flux or helicity is more likely.

Let the spheromak have magnetic flux $\Phi$ enclosed within a volume of size $R$; the magnetic

flux has dimensions $M^{1 / 2} L^{3 / 2} T^{-1}$. The magnetic field on the surface is not constant, but for simplicity we assume that it is of order $B_{0}=\Phi / R^{2}$. Let the external medium have density $\rho_{\text {ext }}$, which has dimensions $M L^{-3}$; the other variable is time whose dimension obviously is $T$. From dimensional analysis we find that expansion of a spheromak obeys a law:

$$
R \sim \rho_{\mathrm{ext}}^{-1 / 6} \Phi^{1 / 3} t^{1 / 3}
$$

A somewhat different approach leading to the same result comes from balancing ram pressure of a strong forward shock, $\rho_{\text {ext }}(d R / d t)^{2}$, with magnetic field energy density on the surface $B^{2} \sim\left(\Phi / R^{2}\right)^{2}$ 
For constant mangetic flux $\Phi$, and assuming that external density scales with radius as $\rho_{\text {ext }} \propto R^{-a}$, the self-similar expansion requires

$$
R \propto t^{2 /(6-a)}
$$

In a constant density medium this gives $R \propto t^{1 / 3}$. Note, that for self-similar scaling the energy in shocked plasma, $\propto R^{2} \rho_{\text {ext }} \dot{R}^{2} \propto t^{-2 /(6-a)}$ decreases with time, as $t^{-1 / 3}$ in constant density, ( $c f$. Farrugia, Osherovich, and Burlaga 1995, Equation (44)). This can be achieved, e.g., if the shocked plasma cools radiatively, but the energy loss should be tuned to the self-similar dynamics, which is not justified.

Thus, generally, if a magnetic bubble drives a non-radiative shock, the surface pressure inside the bubble falls off faster with time than the post-shock pressure and the self-similar assumption breaks down. At every moment the size of the bubble would adjust to the post-shock pressure in a non-self-similar way. For example, if initially the bubble is over pressurized with respect to a surrounding medium of constant density, expansion will drive a shock with $r_{\mathrm{s}} \propto t^{2 / 5}(\operatorname{Sedov} 1959)$. The post-shock pressure scales as $p \propto t^{-6 / 5}$ and the size of the magnetic bubble $R \propto t^{3 / 10}$. Thus, the whole system of magnetic bubble plus external shock medium evolves in a non-self-similar manner, with the relative size of the magnetic bubble decreasing $R / r_{\mathrm{s}} \propto t^{-1 / 10}$. Most energy is transferred to the outside plasma at initial stages of expansion.

\subsection{Expansion in a Wind Environment}

Motion of a CME in a wind is composed of translational motion and overall expansion. The translational velocities of the clouds vary in the range of $300 \mathrm{~km} \mathrm{~s}^{-1}$ to $1000 \mathrm{~km} \mathrm{~s}^{-1}$ with average velocity $478 \mathrm{~km} \mathrm{~s}^{-1}$ (Bothmer and Schwenn 1998). This velocity is of the same order of magnitude as the Solar wind velocity. 11 The local density at the cloud's location is then $\rho_{\mathrm{ext}}=\rho_{0} r_{0}^{2} / r_{\mathrm{CME}}^{2}$, where $r_{\mathrm{CME}} \sim v_{\mathrm{SW}} t$. Substituting in Equation 19 we find that

$$
\begin{aligned}
& R \sim \rho_{0}^{-1 / 6} r_{0}^{-1 / 3} v_{\mathrm{SW}}^{1 / 3} \Phi^{1 / 3} t^{2 / 3} \\
& R \sim \rho_{0}^{-1 / 6} r_{0}^{-1 / 3} v_{\mathrm{SW}}^{-1 / 3} \Phi^{1 / 3} r^{2 / 3} .
\end{aligned}
$$

Bothmer and Schwenn (1998) have found $R \propto r^{0.78 \pm 0.10}$, close to the value of $2 / 3$ predicted by Equation (21).

\footnotetext{
${ }^{1}$ We remark that if we take into account the buoyancy force and the drag due to the motion relative the solar wind we find that the limiting velocity is of the order of the sound speed of the solar wind, which is a few $10^{2} \mathrm{~km} \mathrm{~s}^{-1}$ depending on the temperature which is consistent with the numbers mentioned above.
} 


\section{Observational Signature}

\subsection{Magnetic Field Measured}

The magnetic field measured by a magnetometer as a function of time depends on the relative velocity of the detector and spheromak boundary. Generally, the velocity of the detector with respect to the spheromak's center is dominated by the advection velocity of the spheromak with the Solar wind, which is typically a factor of several larger than velocity of expansion of the spheromak in the winds frame.

Observations of CMEs often indicate that the maximum of B-field is reached before the spacecraft reaches the geometrical center (Burlaga 1995; Farrugia et al. 1993). It was long suspected that is a consequence of the expansion of the magnetic cloud while it moves past the spacecraft (Burlaga 1995, Section 6.5.2): when the detector reaches the middle of the spheromak, the overall normalization of the magnetic field decrease due to expansion. Our model provides a quantitative description of this effect, see Figures 7 and 8.

\subsection{Plasma Velocity}

Observations suggest that the general trend of the velocity measured by a detector crossing the magnetic cloud, is to start from a large value and then decrease (Bothmer and Schwenn 1998) to a smaller but comparable velocity. This is consistent with the image of a magnetic cloud that propagates from the Sun and at the same time expands. We have assumed that the propagation velocity is constant while the expansion is self-similar $R \propto t^{n}$, see Figure 9. In general, clouds differ in the details of the velocity profiles, but most of them give measurements that are in agreement with this model.

\section{Discussion}

In this paper we discuss the self-similar solutions for expanding force-free magnetic structures in spherical and cylindrical geometries. Under the assumption of non-relativistic MHD, when the dynamical effects of the resulting induced electric field can be neglected, the structure remains force-free during expansion, $\mathbf{J}=\alpha \mathbf{B}$. In case of relativistic expansion, when electric field has important dynamical effects (Prendergast 2005; Gourgouliatos and Lynden-Bell 2008), spheromak-type self-similar solutions may be found for constant expansion velocity (R. D. Blandford, private communication). In contrast, our solutions depend

on $R$ and $\dot{R}$ measured at a given time, and thus are applicable to any $R(t)$. 

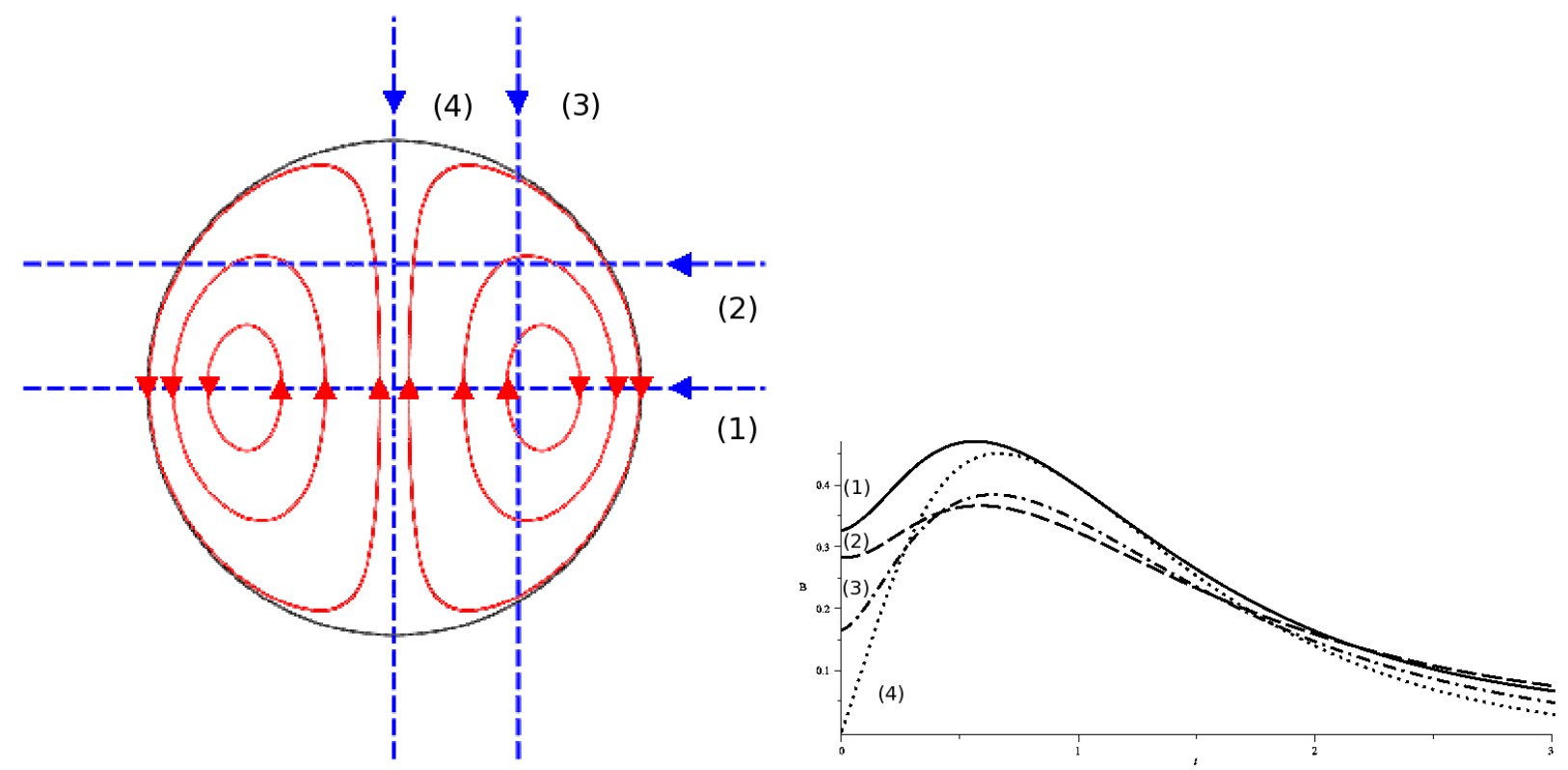

Fig. 7.- Left Panel: Sections of a spheromak with a meridional plane. The trajectories of the detector crossing the spheromak are the dashed lines. Right Panel: Magnetic field measured by a detector flying through an expanding spheromak. The detector enters the cloud at $t=0$, at $t=1$ it crosses the axis: curves (1), (2); or the equator: curves (3), (4). We investigate four cases corresponding to the numbers of the trajectories shown in left panel. The solid curve (1) corresponds to a detector flying on the equator of the spheromak and passing from the centre at $t=1$. The dashed curve (2) corresponds to a detector that flies parallel to the equator and crosses the axis at $t=1$. The dashed-dotted (3) curve corresponds to a detector that flies parallel to the axis and crosses the equator at $t=1$. Finally the dotted curve (4) corresponds to a detector flying along the axis and passing from the centre at $t=1$. In all cases the maximum magnetic field is measured at $t<1$ before the detector crosses the middle of the cloud.

In case of spheromaks, the internal velocity develops azimuthal component, $v_{\phi} \neq 0$, even though the surface expands purely radially. The fact that the magnetic field structure remains force-free during expansion is qualitatively different from the solutions of Low (1982); Farrugia, Osherovich, and Burlaga (1995), which were limited to pure radial motion. As a result, even if at the initial moment magnetic field is force-free, during expansion non-zero pressure forces appeared.

We expect that the solution is dynamically and resistively stable, since stationary forcefree solutions with constant $\alpha$ are very stable configurations (Bellan 2006), both to ideal and resistive instabilities, like tearing mode. Stability of expanding force-free structures 


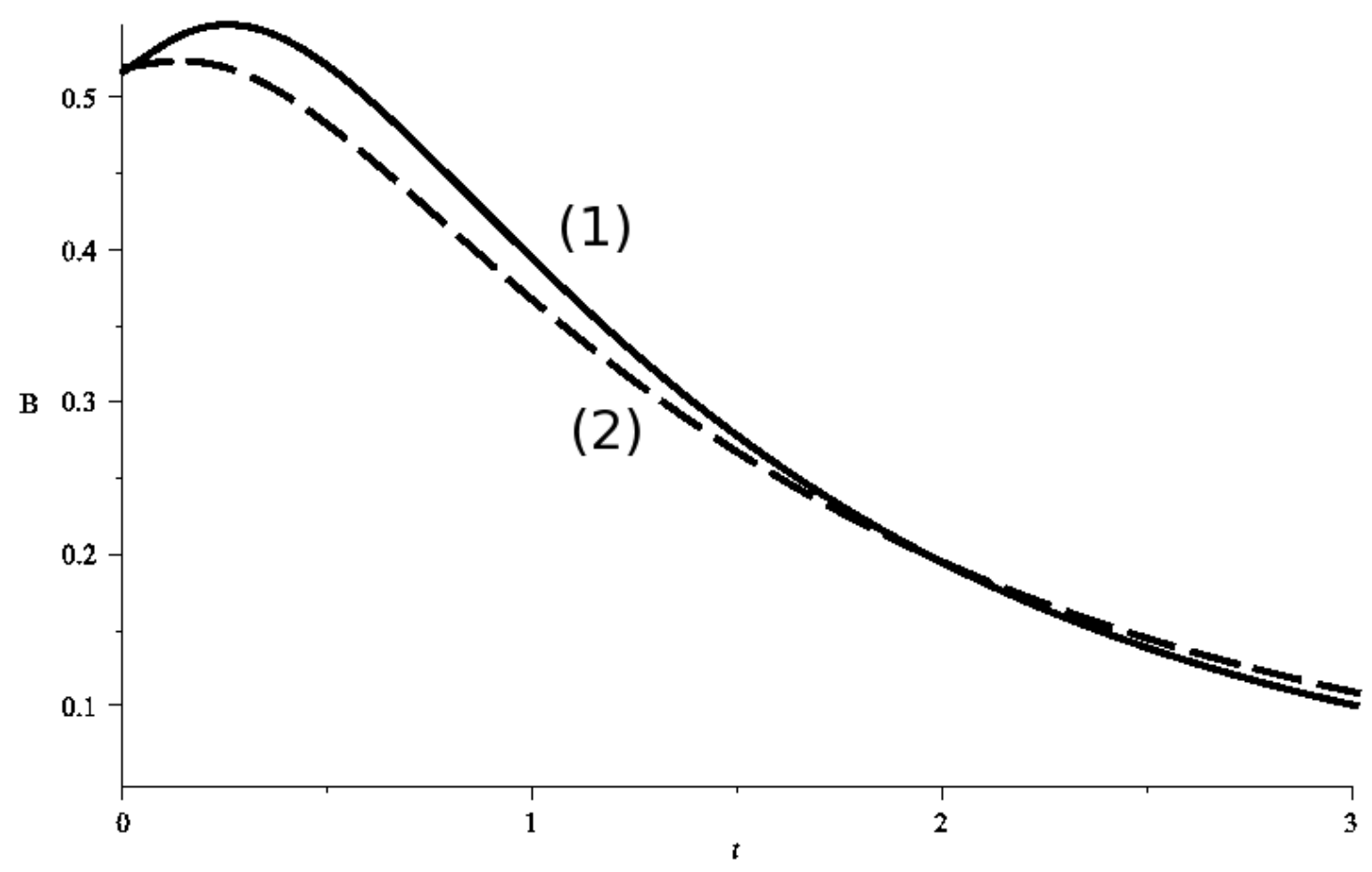

Fig. 8.- Magnetic field measured by a detector flying through an expanding cylinder. The solid curve (1) corresponds to a detector that flies and passes from the centre, whereas the dashed curve (2) correspond to a detector that enters the structure at an impact angle of $\pi / 6$ and misses the centre by half radius.

should be investigated independently, but we expect them to be stable to global modes, since expansion-induced velocity shear will contribute to global stability (but may still result in development of local shearing instabilities).

Expansion of magnetic clouds is qualitative different from the self-similar fluid outflows, since in this case we have an additional constraint, the conservation of magnetic helicity. Since the conservation of magnetic helicity and magnetic energy are, generally, not compatible with each other, the self-similar expansion of spheromak can be connected only to one particular self-similar (Sedov-type) solution, with $r \propto t^{1 / 3}$. This solution has energy decreasing with time and thus requires strong energy losses.

The model predicts that that the maximum magnetic field is reached before the detector reaches geometrical center of a CME, while the plasma velocity measured by the spacecraft smoothly decreases with time. Both these facts are in general agreement with observations. 

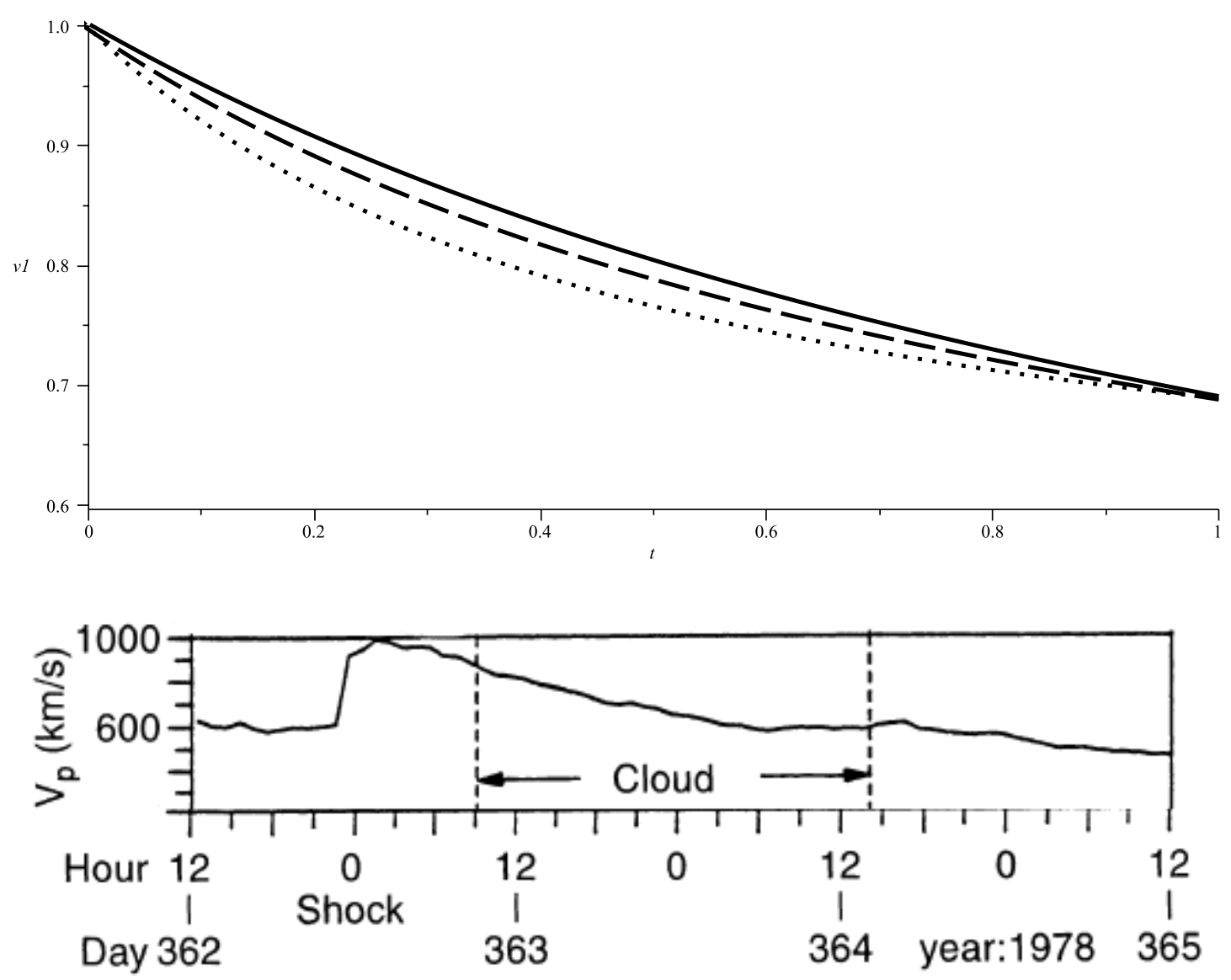

Fig. 9.- Upper panel: The velocity measured by a detector that encounters an expanding magnetic cloud and crosses its axis. These results are valid for both a spheromak cloud provided the detector's trajectory is on the equator and for a cylindrical one provided the trajectory is normal to the axis. We have assumed that the cloud propagates at constant velocity and expands self-similarly so that $R \propto t^{n}$. The detector measures the combined effect. The detector enters the cloud at $t=0$ and exits at $t=1$ where the velocity is 0.7 of the initial. The solid line corresponds to a self-similar expansion with index $n=2 / 3$, the dashed to $n=1 / 2$ and the dotted to $n=1 / 3$. Lower panel: The velocity measured by the Helios of the event on days 362-365 1978 (Bothmer and Schwenn 1998). For $n<1$ the velocity profile in the cloud is consistent with the profile of the expanding model.

\section{Acknowledgements}

We would like to thank Roger Blandford, Charles Farrugia and Martin Laming for insightful discussions. 


\section{REFERENCES}

Bellan, P.M.: 2006, Fundamentals of Plasma Physics, 392.

Bothmer, V., Schwenn, R.: 1998, Annales Geophysicae 16, 1.

Burlaga, L.F.: 1988, J. Geophys. Res. 93, 7217. doi:10.1029/JA093iA07p07217.

Burlaga, L.F.: 1995, Interplanetary magnetohydrodynamics, by L. F. Burlag. International Series in Astronomy and Astrophysics, Vol. 3, Oxford University Press. 1995. 272 pages; ISBN13: 978-0-19-508472-6 3, 89.

Burlaga, L.F., Klein, L., Sheeley, N.R. Jr., Michels, D.J., Howard, R.A., Koomen, M.J., Schwenn, R., Rosenbauer, H.: 1982, Geophys. Res. Lett. 9, 1317. doi:10.1029/GL009i012p01317.

Burlaga, L., Sittler, E., Mariani, F., Schwenn, R.: 1981, J. Geophys. Res. 86, 6673.

Cargill, P.J., Chen, J., Spicer, D.S., Zalesak, S.T.: 1995, Geophys. Res. Lett. 22, 647.

Chandrasekhar, S., Kendall, P.C.: 1957, ApJ 126, 457.

Chen, J., Garren, D.A.: 1993, Geophys. Res. Lett. 20, 2319. doi:10.1029/93GL02426.

Dalakishvili, G., Rogava, A., Lapenta, G., Poedts, S.: 2010, ArXiv e-prints.

Farrugia, C.J., Osherovich, V.A., Burlaga, L.F.: 1995, J. Geophys. Res. 100, 12293.

Farrugia, C.J., Burlaga, L.F., Osherovich, V.A., Richardson, I.G., Freeman, M.P., Lepping, R.P., Lazarus, A.J.: 1993, J. Geophys. Res. 98, 7621. doi:10.1029/92JA02349.

Forbes, T.G., Linker, J.A., Chen, J., Cid, C., Kóta, J., Lee, M.A., Mann, G., Mikić, Z., Potgieter, M.S., Schmidt, J.M., Siscoe, G.L., Vainio, R., Antiochos, S.K., Riley, P.: 2006, Space Science Reviews 123, 251. doi:10.1007/s11214-006-9019-8.

Gourgouliatos, K.N., Lynden-Bell, D.: 2008, MNRAS 391, 268. doi:10.1111/j.13652966.2008.13877.x.

Gourgouliatos, K.N., Vlahakis, N.: 2010, ArXiv e-prints.

Ivanov, K.G., Kharshiladze, A.F.: 1985, Sol. Phys. 98, 379. doi:10.1007/BF00152467.

Ivanov, K.G., Harshiladze, A.F., Eroshenko, E.G., Stiazhkin, V.A.: 1989, Sol. Phys. 120, 407. 
Klein, L.W., Burlaga, L.F.: 1982, J. Geophys. Res. 87, 613. doi:10.1029/JA087iA02p00613.

Lepping, R.P., Burlaga, L.F., Jones, J.A.: 1990, J. Geophys. Res. 95, 11957. doi:10.1029/JA095iA08p11957.

Low, B.C.: 1982, ApJ 254, 796. doi:10.1086/159790.

Lundquist, S.: 1951, Physical Review 83, 307. doi:10.1103/PhysRev.83.307.

Miller, G., Turner, L.: 1981, Physics of Fluids 24, 363. doi:10.1063/1.863351.

Prendergast, K.H.: 2005, MNRAS 359, 725. doi:10.1111/j.1365-2966.2005.08949.x.

Sedov, L.I.: 1959, Similarity and Dimensional Methods in Mechanics, 244.

Taylor, J.B.: 1974, Physical Review Letters 33, 1139. doi:10.1103/PhysRevLett.33.1139.

Vandas, M., Fischer, S., Geranios, A.: 1991, Planet. Space Sci. 39, 1147. doi:10.1016/00320633(91)90166-8.

Vandas, M., Fischer, S., Pelant, P., Dryer, M., Smith, Z., Detman, T.: 1997, J. Geophys. Res. 102, 24183. doi:10.1029/97JA02257.

Woltjer, L.: 1958, ApJ 128, 384. 